\title{
Analysis of the vaccine-induced influenza B virus hemagglutinin-specific antibody dependent cellular cytotoxicity response
}

\author{
Rory D. de Vries ${ }^{\mathrm{a}}$, Nella J. Nieuwkoop ${ }^{\mathrm{a}}$, Florian Krammer ${ }^{\mathrm{b}}$, Branda $\mathrm{Hu}^{\mathrm{c}}$, \\ Guus F. Rimmelzwaan ${ }^{\mathrm{a}, 1, *}$ \\ a Department of Viroscience, Erasmus MC, Rotterdam, the Netherlands \\ ${ }^{\mathrm{b}}$ Department of Microbiology, Icahn School of Medicine at Mount Sinai, New York, NY, USA \\ ${ }^{c}$ Global Clinical Immunology Department, Sanofi Pasteur, Swiftwater, PA, USA
}

\section{A R T I C L E I N F O}

\section{Keywords:}

Influenza_B_Virus

Hemagglutinin

Antibodies

Natural_Killer_Cells

Antibody-Dependent_Cellular_Cytotoxicity

\begin{abstract}
A B S T R A C T
Influenza A virus (IAV) and influenza B virus (IBV) cause substantial morbidity and mortality during seasonal epidemics. On basis of variation in the surface glycoprotein hemagglutinin, two antigenically distinct lineages of IBV are distinguished: B/Victoria/2/87-like (B/Vic) and B/Yamagata/16/88-like (B/Yam). To prevent IAV and IBV infections, both trivalent (containing IBV of one lineage) and quadrivalent (containing IBV of both lineages) influenza vaccines are used. In addition to virus-neutralizing antibodies, inactivated influenza vaccines induce antibodies that mediate antibody-dependent cellular cytotoxicity (ADCC). Here, we determine whether vaccination with trivalent or quadrivalent inactivated influenza vaccine induces ADCC mediating antibodies directed to IBV of the two different lineages, and whether these antibodies cross-react with IBV of the opposing lineage. A robust ADCC assay based on the use of recombinant hemagglutinin and a continuous natural killer cell line that expresses Fc $\gamma$ RIII (CD16) was used to detect the presence of ADCC mediating antibodies. Paired pre- and postvaccination serum samples from 26 and 15 study subjects that received a trivalent or quadrivalent inactivated influenza vaccine, respectively, were assessed for the presence of ADCC mediating antibodies specific for HA derived from viruses of the B/Vic or B/Yam-lineage. Furthermore, the relative contribution of HA1- and HA2subunit-specific antibodies to the ADCC response was determined. We found that seasonal inactivated influenza vaccines induce HA-head- and HA-stalk-specific antibodies that mediate ADCC. As expected, the quadrivalent vaccine induced antibodies to HA from both IBV lineages. Notably, a trivalent vaccine containing HA from the B/ Vic lineage induced antibodies that cross-react with the B/Yam lineage.
\end{abstract}

\section{Introduction}

Influenza viruses cause $3-5$ million cases of severe illness and 290,000-650,000 deaths per year worldwide during annual epidemics (Lambert and Fauci, 2010). Co-circulating influenza A viruses (IAV) and influenza B viruses (IBV) are responsible for this morbidity and mortality. Similarly to IAV, IBV accumulates mutations in the gene encoding the surface glycoprotein hemagglutinin (HA) to avoid recognition by neutralizing antibodies induced by previous vaccinations or infections, a process known as antigenic drift (Smith et al., 2004). Based on genetic and antigenic variation of HA, two antigenically distinct lineages of IBV are distinguished: B/Victoria/2/87-like (B/Vic) and B/ Yamagata/16/88-like (B/Yam) (Rota et al., 1990).
Currently used inactivated influenza vaccines aim at the induction of virus-neutralizing antibodies directed to the variable head domain of HA. These vaccines are trivalent (TIV) or quadrivalent (QIV) and contain IBV antigens of one or both lineages, respectively. However, antigenic mismatches between circulating viruses and strains used for TIV production frequently occur (Ambrose and Levin, 2012) and consequently, TIV-induced antibodies neutralize IBV of the opposing lineage inefficiently (Bodewes et al., 2011; van de Sandt et al., 2015). The difficulty of predicting which IBV lineage will circulate in the next influenza season and poor cross-neutralization of IBV-specific antibodies is the basis for the rationale to include IBV of both lineages in the vaccine.

Virus neutralizing antibodies are mainly directed to antigenic sites

\footnotetext{
*Corresponding author at: Alexander von Humboldt Professor in Virology, University of Veterinary Medicine, Research Centre for Emerging Infections and Zoonoses, Bünteweg 17, 30559 Hannover, Germany.

E-mail address: Guus.rimmelzwaan@tiho-hannover.de (G.F. Rimmelzwaan).

${ }^{1}$ Current address: University of Veterinary Medicine Hannover, Hannover, Germany.
} 
A

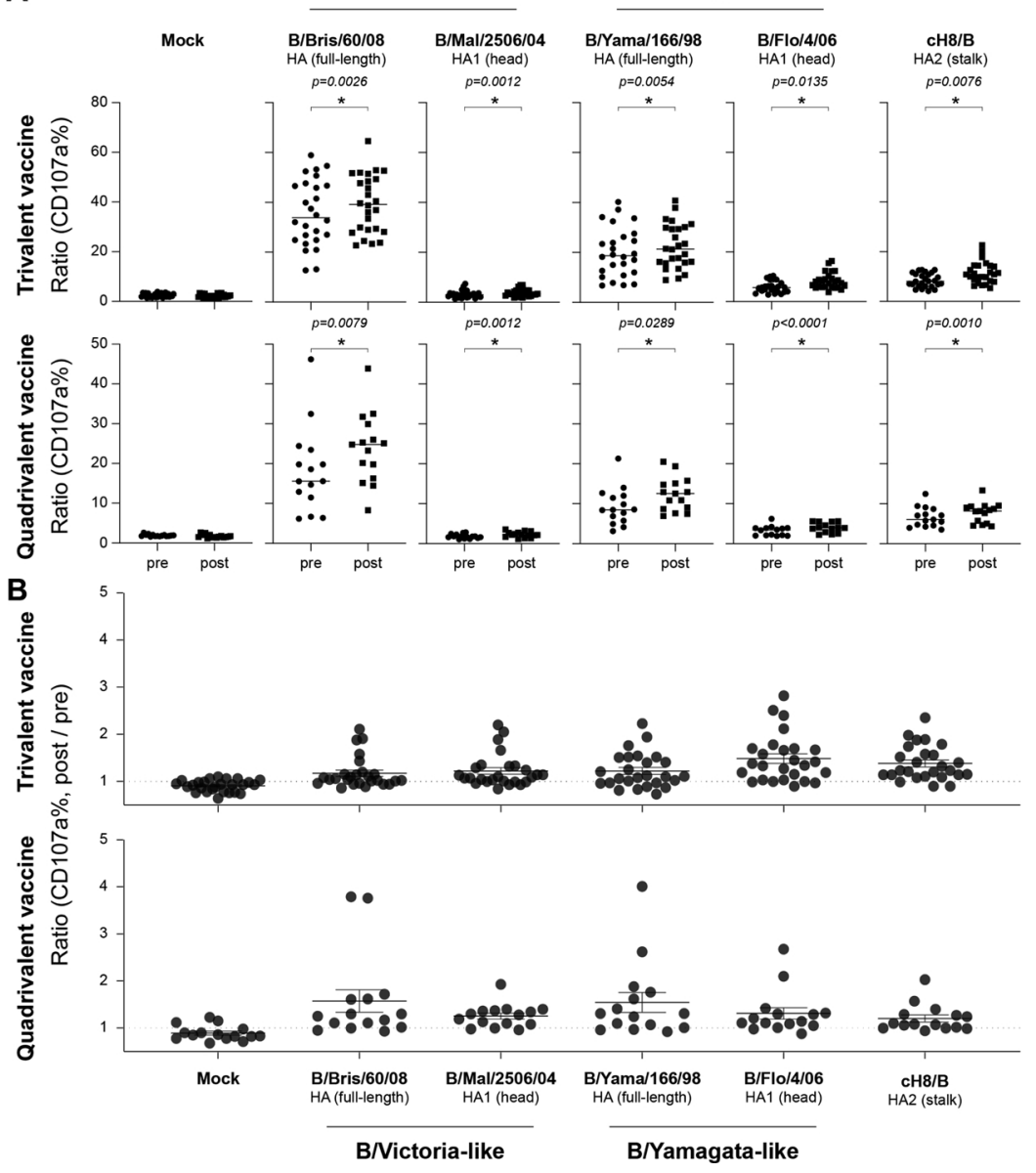

Fig. 1. Induction of ADCC mediating antibodies by vaccination with QIV or TIV. Presence of ADCC mediating antibodies was determined by a flow cytometry-based assay performed with paired serum samples obtained from 15 study subjects that received QIV and 26 study subjects that received TIV. Sera were tested against a full-length HA from B/Brisbane/60/08 (B/Vic lineage) and $\mathrm{B} /$ Yamanashi/166/98 (B/Yam lineage), the HA1 subunits of B/Malaysia/2506/04 B/Vic lineage) and B/Florida/4/ 06 (B/Yam lineage), and a chimeric HA consisting of the stalk of B/Yamagata16/88 and the head domain of an IAV of the H8 subtype (cH8/B). (A) percentages obtained with pre- and postvaccination samples are plotted for comparison. (B) Response ratios were calculated by dividing CD107a percentages in post-vaccination samples by CD107a percentages pre-vaccination. A ratio $>1$ means a positive response to vaccination. in or around the receptor-binding site located in the head domain of HA. In addition to these, antibodies with other immunologically important modes of action are induced. It has been demonstrated that both natural infection and vaccination induce antibodies that mediate antibody-dependent cellular cytotoxicity (ADCC), in addition to virus neutralizing (VN) antibodies (de Vries et al., 2017a, b). Since these ADCC mediating antibodies generally target the HA-stalk or conserved regions in the HA-head, they are likely to display a high degree of crossreactivity and may be a correlate of cross-protective immunity (Asthagiri Arunkumar et al., 2019; DiLillo et al., 2014). Therefore, the HA-stalk is generally considered as an important target for future, more broadly protective influenza vaccines.

Here, we determined whether vaccination with TIV or QIV induces ADCC mediating antibodies directed to HA of IBV belonging to the two different lineages. Furthermore, we analysed whether these antibodies are specific for the HA-head or -stalk and whether these antibodies have the capacity to cross-react with IBV of the opposing lineage.

\section{Materials \& methods}

\subsection{Ethics statement}

The work described here has been carried out in accordance with the code of ethics of the world medical association (declaration of Helsinki). Informed consent was obtained from all participants. Samples were obtained from clinical trials identified as NCT01946438 and NCT02258334 (clinicaltrials.gov).

\subsection{Serum samples}

Pre- and post-vaccination paired sera obtained from 41 healthy study subjects (18 years up to 65, and 65 and older) were assessed for the presence of ADCC mediating antibodies. Twenty-six subjects received TIV (A/California/07/2009 (H1N1pdm09), A/Texas/50/2012 (H3N2) and B/Massachusetts/02/2012 [B/Yam-lineage]) and fifteen subjects received QIV (A/California/07/2009, A/Texas/50/2012, B/ Massachusetts/02/2012 [B/Yam-lineage] and B/Brisbane/60/2008 [B/ Vic-lineage]). Vaccines used were split inactivated influenza vaccines from the 2013-2014 and 2014-2015 season, containing $15 \mu \mathrm{g}$ HA per subtype. HI titers of these sera had been determined as an endpoint of the clinical trials, but could not be unblinded at the time of preparation of this manuscript. Consequently, serum samples were selected obtained from individuals that displayed a wide range of $\mathrm{HI}$ antibody responses to vaccination.

\subsection{ADCC assay}

ADCC assays were performed as described previously (de Vries et al., 2017a, b). Briefly, either purified full-length baculovirus expressed recombinant HA protein or the HA1 subunit, were used. Fulllength HA from B/Brisbane/60/08 and HA1 from B/Malaysia/2506/04 were used as representative antigens from the $\mathrm{B} / \mathrm{Vic}$ lineage, whereas full-length HA from B/Yamanashi/166/98 and HA1 from B/Florida/4/ 06 were used as B/Yam representatives. To test whether stalk-specific antibodies were induced by vaccination, a chimeric full-length antigen was used, consisting of the (irrelevant) head domain of an IAV of the H8 subtype (A/mallard/Sweden/24/02) and the stalk of IBV B/Yamagata/ 16/88 (cH8/B). Microtiter plates (high-binding 96-wells flat bottom, 
Immunolon) were coated overnight (200 ng/well). After coating, plates were washed with PBS, blocked with $5 \%$ bovine serum albumin (BSA) and $100 \mu \mathrm{l}$ volumes of diluted (1:160) serum sample was added. Negative and positive control serum sample were included in all experiments. All sera were tested in duplicate, the complete assay was fully reproduced twice. The continuous NK cell-line NK92.05-CD16 was used as source of NK cells (Binyamin et al., 2008) and the presence of ADCC mediating antibodies specific for the respective antigens was detected by analysing these NK cells for the expression of degranulation marker CD107a by flow cytometry. Live cells were gated on basis of forward and sideward scatter and the percentage of CD107a positive cells was acquired.

\subsection{Statistical analysis}

Statistical analyses were performed using GraphPad Prism. $\mathrm{CD}_{107 \mathrm{a}^{+}}$percentages obtained with pre- and post-vaccination sera were compared by performing a $t$-test on paired samples when the data was normally distributed, or a Wilcoxon rank test on paired samples when the data did not pass normality testing (Fig. 1A). Response ratios were calculated by dividing CD107a percentages in post-vaccination samples by CD107a percentages pre-vaccination. A ratio $>1$ is indicative of a positive response to vaccination (Fig. 1B)

\section{Results}

\subsection{QIV induces ADCC mediating antibodies}

The fifteen study subjects that received QIV developed significant ADCC mediating antibody responses to the full-length HA from both IBV lineages (B/Vic: $p=0.0026$, B/Yam: $p=0.0054$ ) (Fig. 1A and B). Additionally, modest, but statistically significant ADCC mediating antibody responses to the HA1 subunit of both lineages were detected (B/ Vic: $p=0.0012$, B/Yam: $p=0.0135$ ), as well as responses to the HAstalk (cH8/B, $p=0.0076)$ (Fig. $1 \mathrm{~A}$ and $\mathrm{B})$.

\subsection{TIV induces cross-lineage ADCC mediating antibodies}

The twenty-six study subjects that received TIV, containing IBV from the B/Yam lineage (B/Massachusetts/02/2012), developed significant ADCC mediating antibody responses to both the full-length HA of the matching lineage (B/Yamanashi/166/98, $p=0.0289)$ and the full-length HA of the opposing lineage (B/Brisbane/60/08, $p=0.0079$ ) (Fig. 1A and B). Notably, although HA1-specific ADCC mediating antibodies were detected at a low level, a statistically significant response to HA1 of the matching (B/Flo/4/06, $p<0.0001)$ and opposing (B/ $\mathrm{Mal} / 2506 / 04, p=0.0012$ ) lineage was also detected (Fig. 1A and B). Additionally, an IBV HA-stalk-specific ADCC mediating antibody response was observed after vaccination with TIV $(p=0.0010)$.

\section{Discussion}

Although hemagglutination inhibition (HI) and virus neutralizing (VN) antibodies are regarded as the major correlates of protection from (re-)infection with antigenically matching influenza virus strains, nonneutralizing antibodies can also contribute to protective immunity. It has previously been demonstrated that infection with IAV and IBV leads to the induction of ADCC mediating antibodies (de Vries et al., 2017a, b; Mesman et al., 2016), and vaccination of subjects with pre-existing immunity to influenza viruses can increase the levels of ADCC mediating antibodies (de Vries et al., 2017a). It was also shown that primary IBV infection led to the formation of cross-lineage ADCC mediating antibodies, i.e. antibodies reactive with IBV from the opposing lineage (de Vries et al., 2017b). Here, we show that administration of inactivated influenza vaccines induces IBV-specific ADCC mediating antibody responses. As expected, the use of QIV induced ADCC mediating antibodies reactive with $\mathrm{HA}$ of viruses belonging to both the $\mathrm{B} / \mathrm{Vic}$ and $\mathrm{B} /$ Yam lineage. Notably, the use of TIV containing IBV of the B/Yam lineage only, induced ADCC mediating antibodies to viruses of the matching and the opposing lineage. This indicates that these antibodies are cross-reactive and recognize conserved epitopes in both the head and stalk domain.

Recent studies show that antibodies targeting the receptor-binding domain located in the HA-head are not capable of mediating ADCC (Asthagiri Arunkumar et al., 2019; DiLillo et al., 2014; Cox et al., 2016; He et al., 2016; Leon et al., 2016). A stabilized immunological synapse between target and effector cell is required for ADCC activity, facilitated by interactions between the receptor-binding domain of HA and sialic acids on the one hand, and the Fc part of virus-specific immunoglobulin molecules and the Fc $\gamma$ RIII on the other. Here, we show that inactivated vaccines induce ADCC mediating antibodies specific for both the HA1 and HA2 subunits. Notably, the HA1 subunit used in the assays here is mainly the HA-head, but also contains part of the HAstalk. HA1-specific antibodies, likely reflecting HA-head-specific antibodies, were only detected at low levels and were most likely specific for epitopes located outside the receptor-binding domain. We therefore confirmed that in the solid-phase ADCC assay and with the use of NK92.05-CD16 cells, 2 points of contact between effector cells and HA are still required for ADCC activity. Since epitopes located in these regions are generally more conserved, this potentially explains the cross-lineage reactivity of the vaccine induced ADCC antibodies.

In conclusion, we show that with the use of seasonal inactivated influenza vaccines, IBV-specific ADCC mediating antibodies specific for both the HA-head and HA-stalk were induced. In addition, TIV containing HA derived from the B/Yam lineage induced antibodies crossreactive with the $\mathrm{B} / \mathrm{Vic}$ lineage.

\section{Transparency document}

The Transparency document associated with this article can be found in the online version.

\section{CRediT authorship contribution statement}

Rory D. de Vries: Conceptualization, Data curation, Writing - original draft. Nella J. Nieuwkoop: Methodology, Formal analysis. Florian Krammer: Methodology, Writing - review \& editing. Branda Hu: Resources, Data curation, Writing - review \& editing. Guus F. Rimmelzwaan: Conceptualization, Writing - review \& editing, Supervision.

\section{Acknowledgements}

The authors thank Dr. Kerry S. Campbell for supplying the NK92.06CD16 cell line. This work was supported by Innovative Medicines Initiative (IMI) grant FLUCOP [115672-3]. Furthermore, financial support was received from EU grant FLUNIVAC [602604, GFR and RDdV]. The funding source had no role in study design, or collection, analysis and interpretation of the data. No competing interests are declared. Serum samples were provided by Sanofi Pasteur.

\section{References}

Ambrose, C.S., Levin, M.J., 2012. The rationale for quadrivalent influenza vaccines. Hum. Vaccin. Immunother. 8 (1), 81-88.

Asthagiri Arunkumar, G., et al., 2019. Broadly cross-reactive, nonneutralizing antibodies against influenza B virus hemagglutinin demonstrate effector function-dependent protection against lethal viral challenge in mice. J. Virol. 93 (6).

Binyamin, L., et al., 2008. Blocking NK cell inhibitory self-recognition promotes antibodydependent cellular cytotoxicity in a model of anti-lymphoma therapy. J. Immunol. 180 (9), 6392-6401.

Bodewes, R., et al., 2011. Prevalence of antibodies against seasonal influenza A and B viruses in children in Netherlands. Clin. Vaccine Immunol. 18 (3), 469-476.

Cox, F., et al., 2016. HA antibody-mediated FcgammaRIIIa activity is both dependent on 
FcR engagement and interactions between HA and sialic acids. Front. Immunol. 7, 399.

de Vries, R.D., et al., 2017a. Influenza virus-specific antibody dependent cellular cytoxicity induced by vaccination or natural infection. Vaccine 35 (2), 238-247.

de Vries, R.D., et al., 2017b. Primary human influenza B virus infection induces crosslineage hemagglutinin stalk-specific antibodies mediating antibody-dependent cellular cytoxicity. J. Infect. Dis. 217 (1), 3-11.

DiLillo, D.J., et al., 2014. Broadly neutralizing hemagglutinin stalk-specific antibodies require FcgammaR interactions for protection against influenza virus in vivo. Nat. Med. 20 (2), 143-151.

He, W., et al., 2016. Epitope specificity plays a critical role in regulating antibody-dependent cell-mediated cytotoxicity against influenza A virus. Proc. Natl. Acad. Sci. U. S. A 113 (42), 11931-11936.

Lambert, L.C., Fauci, A.S., 2010. Influenza vaccines for the future. N. Engl. J. Med. 363
(21), 2036-2044.

Leon, P.E., et al., 2016. Optimal activation of Fc-mediated effector functions by influenza virus hemagglutinin antibodies requires two points of contact. Proc. Natl. Acad. Sci. U. S. A. 113 (40), E5944-E5951.

Mesman, A.W., et al., 2016. Influenza virus A(H1N1)2009 antibody-dependent cellular cytotoxicity in young children prior to the H1N1 pandemic. J. Gen. Virol. 97 (9), 2157-2165.

Rota, P.A., et al., 1990. Cocirculation of two distinct evolutionary lineages of influenza type B virus since 1983. Virology 175 (1), 59-68.

Smith, D.J., et al., 2004. Mapping the antigenic and genetic evolution of influenza virus. Science 305 (5682), 371-376.

van de Sandt, C.E., et al., 2015. Influenza B viruses: not to be discounted. Future Microbiol. 10 (9), 1447-1465. 